\title{
Multiplicity of Very Massive Stars
}

\author{
M. Heydari-Malayeri \\ European Southern Observatory \\ La Silla, Chile
}

Abstract. Are there very massive stars (VMSs) of mass greater than $100 M_{\odot}$ ? This question constitutes one of the fundamental problems of astrophysics. We present observational evidence against the existence of such stars in the Magellanic Clouds. The multiplicity of VMSs has several important consequences for astrophysics. If VMSs do not exist we need to revise our ideas about the formation and evolution of stars.

The question of the upper mass limit is very important for star formation theories and its related subjects, for example the initial mass function (IMF). The multiplicity of massive star alters the IMF in two ways. It eliminates the stars more massive than about $100 M_{\odot}$ and at the same time brings about a large number of stars in the mass interval $30-60 M_{\odot}$. This has important implications for several astrophysical problems, e.g. the evolution of galaxies and the choice of extragalactic distance indicators.

Today in spite of recent theoretical progress made in understanding the formation and evolution of massive stars the question of exact upper limit to stellar masses remains open. In this regard the problem with R136, once believed to have a mass of $3000 M_{\odot}$ is not fully resolved, since its main component may be about $250 M_{\odot}$ (Walborn, 1984). But can stars of this mass exist? Observational results are of vital importance in answering this vital question.

We summarize in Table 1 some results on the previously supposed supermassive stars in the Magellanic Clouds. The observations were carried out using the ESO $2.2 \mathrm{~m}$ and NTT telescopes. The observations and image processing methods are explained in Heydari-Malayeri et al. $(1988,1989)$ and Heydari-Malayeri and Hutsemékers (1990).

The global spectral type and visual magnitudes of the stars are given in columns 2 and 3 respectively. The mass previously attributed to each star is listed in column 4. The number of resolved components is presented in column 5. The $V$ magnitude of the brightest component is given in column 6 and the corresponding mass in column 7 . The masses were estimated using the new evolutionary models of massive stars for the Magellanic Clouds metallicities (Maeder, 
1990).

Table 1. New mass estimates

\begin{tabular}{|lccccccc|}
\hline Star & $S p$. & $V_{p}$ & $(\text { ZAMS })_{p}$ & Components & $V_{n}$ & (ZAMS $_{n}$ & Notes \\
\hline LMC & & & & & & & \\
Sk-66 41 & O5 V & 11.72 & $>120$ & 6 & 12.2 & 85 & (a) \\
Sk-69 253 & $\begin{array}{c}\text { O9.5: I } \\
\text { or B 0.7-1 I }\end{array}$ & 11.23 & 120 & 14 & 12.0 & 60 & \\
& & & & & & & \\
SMC & & & & & & & \\
Sk-157 & O9.5: III & 12.17 & 60 & 11 & 13.2 & 40 & \\
NGC 346 \#1 & O4 & 12.43 & 130 & 3 & 12.3 & 85 & (b) \\
NGC 346 \#2 & O3 & 13.43 & 90 & 1 & - &.. & (b) \\
\hline
\end{tabular}

(a) Main component probably multiple

(b) See Heydari-Malayeri \& Hutsemékers (1990)

New results on several other so-called VMSs will be presented in a forthcoming paper.

\section{References}

Heydari-Malayeri, M., Magain, P., Remy, M.: 1988, Astron. Astrophys. 201, L41

Heydari-Malayeri, M., Magain, P., Remy, M.: 1989, Astron. Astrophys. 222, 41

Heydari-Malayeri, M., Hutsemékers, D.: 1990, in preparation

Maeder, A.: 1990, Astron. Astrophys. Suppl. Ser., in press

Walborn, N.R.: 1984, in Structure and Evolution of the Magellanic Clouds,

IAU Sympos. 108, eds. S. van den Bergh, K.S. de Boer, p. 243 